CCNY-HEP-96/1

IUHET 326

January 1996

hep-ph/9610399

\title{
Nonequilibrium Neutrino Oscillations in the Early Universe with an Inverted Neutrino-Mass Hierarchy
}

\author{
V. Alan Kosteleckýa and Stuart Samuel ${ }^{b *}$ \\ ${ }^{a}$ Physics Department \\ Indiana University \\ Bloomington, IN 47405, U.S.A. \\ ${ }^{b}$ Physics Department \\ City College of New York \\ New York, NY 10031, U.S.A.
}

\begin{abstract}
The annihilation of electron-positron pairs around one second after the big bang distorts the Fermi-Dirac spectrum of neutrino energies. We determine the distortions assuming neutrino mixing with an inverted neutrino-mass hierarchy. Nonequilibrium thermodynamics, the Boltzmann equation, and numerical integration are used to achieve the results. The various types of neutrino behavior are established as a function of masses and mixing angles.
\end{abstract}

*Electronic address: samuel@scisun.sci.ccny.cuny.edu 
Around a tenth of a second after the big bang, the early Universe was predominantly a hot lepton-photon gas in thermal equilibrium [1]. Shortly thereafter, when the temperature of the Universe fell to about one $\mathrm{MeV}$, the electrons and positrons annihilated into photons. The transfer of the lepton energy to the photon gas made the photon temperature slightly higher than if the lepton annihilation had not occurred. At about the same time, the neutrinos decoupled thermally because the frequency of hard-scattering processes decreased due to the lower neutrino energies.

Since electron neutrinos couple more strongly to electrons and positrons than muon or tau neutrinos, the electron neutrinos received more energy from the electronpositron annihilations. In addition, higher-energy neutrinos decoupled later than lower-energy ones. As a result, distortions appeared in the neutrino Fermi-Dirac distributions. The timing of the neutrino decoupling is critical for the distortions to arise. If the neutrinos had decoupled much earlier or much later than the electronpositron annihilation, then little or no distortion of thermodynamic distributions would have arisen. One consequence of the timing coincidence is that an excess of $\nu_{e}$ over $\nu_{\mu}$ and $\nu_{\tau}$ formed around this time. The distortions have been analyzed in the case of no neutrino mixing in refs. [2, 3].

The issue of how the standard model of the early Universe is affected by the presence of various types of neutrino and of neutrino masses and mixings has been investigated by several authors during the past few years [4]-[16]. In the present work, we assume that only electron and muon neutrinos have significant mixing. Then, on average no neutrino oscillations occur in the early Universe before the $\nu_{e}$ excess develops, because until then for every $\nu_{e}$ that oscillates into a $\nu_{\mu}$ there is a $\nu_{\mu}$ that oscillates into a $\nu_{e}$. However, neutrino mixing can affect the generation of the spectral distortions [16]. Also, after the neutrinos decouple and the distortions are established, energies and densities continue to fall as the Universe expands and so the relevance of neutrino mixing increases [11, 14].

The neutrino mixing can be characterized by the vacuum neutrino-mixing angle $\theta$ and the difference $\Delta=m_{2}^{2}-m_{1}^{2}$ between the squared masses of the mass eigenstates. The parameter region $\Delta<0$ corresponds to an inverted neutrino-mass hierarchy, in which the heavier mass eigenstate has a larger component in the electron neutrino 
than in the muon neutrino. For $\Delta<0$, the flavor evolution of neutrinos in the early Universe exhibits a rich spectrum of features [12]. Several recent papers have been based on the possibility of an inverted neutrino-mass hierarchy [17, 18, 19].

For $|\Delta|<10^{-6} \mathrm{eV}^{2}$, neutrino mixing effects are too small to affect significantly the production of the $\nu_{e}$ excess, so neutrino oscillations are relevant only after production. It is therefore a good approximation to assume the production phase, $t<0.33$ seconds, generates the known zero-mixing distortions [2] and to adopt the results as input for the oscillation phase [11, 12, 14]. This procedure avoids the difficulties of simultaneously treating the nonequilibrium neutrino-excess production and the neutrino oscillations.

In the region $10^{-6} \mathrm{eV}^{2}<|\Delta|<10^{-5} \mathrm{eV}^{2}$, oscillations play some role in the distortion of neutrino distributions, while for $|\Delta|>10^{-5} \mathrm{eV}^{2}$ oscillations definitely must be incorporated in the excess production process. For $\Delta>10^{-6} \mathrm{eV}^{2}$, another approximation is available to bypass the complexities of the full production-oscillation process: the appropriate initial conditions for the pure-oscillation phase can be estimated by projecting the zero-mixing neutrino excess onto approximate nonlinear mass-eigenstate (ANME) configurations [12, 14]. It has recently been demonstrated that ANME configurations are indeed the correct initial conditions for the oscillation phase for the case of positive $\Delta>10^{-6} \mathrm{eV}^{2}$ [16.

To date, no analysis of neutrino oscillations in the early Universe has been performed for negative $\Delta<-10^{-6} \mathrm{eV}^{2}$. The basic reason is that early-Universe neutrino oscillations are unstable when $\Delta$ is negative, and so at some point in time the average neutrino flavor undergoes a dramatic change in behavior. This time is called the instability time in what follows. When $-10^{-6} \mathrm{eV}^{2}<\Delta<0$, the instability time occurs after the production phase, and hence does not affect the neutrino excess. The method of ANME configurations can therefore be used [12]. However, for $\Delta<-10^{-6}$ $\mathrm{eV}^{2}$, the instability time occurs during the production phase, which makes it difficult to surmise appropriate initial conditions for the subsequent neutrino oscillations.

The primary purpose of the present work is to analyze this remaining unexplored parameter region. Using the full apparatus for the analysis of combined oscillations and production, we have obtained results for the neutrino spectral distortions and 
for neutrino oscillations for $\Delta<-10^{-6} \mathrm{eV}^{2}$. A secondary purpose is to verify the validity of the ANME-based initial conditions assumed in ref. [12] for the oscillation phase in the region $-10^{-6} \mathrm{eV}^{2}<\Delta<0$.

The lengthy equations governing the excess- $\nu_{e}$ production in the presence of oscillations may be found in section $\mathrm{V}$ of ref. [16]. They are based on nonequilibrium thermodynamics using the Boltzmann equation, and they incorporate hard-scattering production effects from interactions among photons, electrons, positrons, and neutrinos, as well as neutrino forward scattering off neutrinos, electrons, and positrons. Neutrino-neutrino forward scattering is often the dominant neutrino-oscillation effect by many orders of magnitude. It renders the system of equations nonlinear, so numerical simulations are necessary. In the present work, we employ the notation and methods of ref. [16. In particular, $v_{0}(E, t) \equiv\left(\nu_{e}^{\dagger} \nu_{e}+\nu_{\mu}^{\dagger} \nu_{\mu}\right)$ is the sum of the excess of electron and muon neutrinos at energy $E$ per canonical comoving volume per $E / T$, $v_{1}(E, t) \equiv\left(\nu_{e}^{\dagger} \nu_{e}-\nu_{\mu}^{\dagger} \nu_{\mu}\right)$ is the difference, $v_{4}(E, t) \equiv \nu_{\tau}^{\dagger} \nu_{\tau}$ is the excess of tau neutrinos, while $v_{2}(E, t) \equiv 2 \operatorname{Re}\left(\nu_{e}^{\dagger} \nu_{\mu}\right)$ and $v_{3}(E, t) \equiv 2 \operatorname{Im}\left(\nu_{e}^{\dagger} \nu_{\mu}\right)$ are the real and imaginary parts of the off-diagonal component of the density matrix for neutrinos with energy $E$. In units with $\hbar=c=1$, the canonical comoving volume is taken to be $1.0 \mathrm{MeV}^{-3}$ when the temperature $T$ of the Universe is $1.5 \mathrm{MeV}$.

For positive $\Delta$, a single behavior characterizes neutrino flavor evolution [14]: although the flavor may change over time, any evolution occurs smoothly. In contrast, when $-10^{-6} \mathrm{eV}^{2}<\Delta<0$ three types of behavior appear [12 depending on the values of $\theta$ and $\Delta$. Near $\sin ^{2} 2 \theta=1$, the evolution is smooth. For $\sin ^{2} 2 \theta$ small, selfmaintained coherence occurs. This is a mode exhibiting some features reminiscent of solitons, in which a fraction of the neutrinos oscillate essentially in phase. It was originally observed in test simulations [20] and is now understood mathematically [21]. The third behavior, called irregular in ref. [12], occurs for intermediate $\sin ^{2} 2 \theta$ and relatively large $|\Delta|$.

Mathematical and physical insight about irregular behavior has recently been obtained [22]. Ignoring the expansion of the Universe but incorporating the alignment property of the neutrino vectors [12, 14], analytical solutions for the behavior of the average neutrino flavor were found. These solutions are characterized by two modes. 
When both have very small amplitude, smooth behavior occurs. When only one mode has sizeable amplitude, self-maintained coherence arises. Finally, when both modes are significantly excited, bimodal self-maintained coherence appears. This can mimic the irregular behavior seen in ref. [12]. It has been conjectured that, when the periods of the two modes are incomensurate, bimodal self-maintained coherence is irregular behavior [22].

To verify that incorporating hard-scattering production effects leaves unaffected the results of ref. [12], we performed full production-oscillation simulations for selected values of $\sin ^{2} 2 \theta$ and $\Delta$ in the region $-10^{-6} \mathrm{eV}^{2}<\Delta<0$. These simulations qualitatively confirm the results of ref. [12] throughout the entire parameter region. They also agree quantitatively over all regions displaying self-maintained coherence or smooth evolution. For example, Figures 2 and 4 of ref. [12] are reproduced. In the irregular region, the numerical results are qualitatively the same, and the quantitative discrepancy can be understood in terms of bimodal coherence. It turns out that the effect of incorporating the true neutrino-excess production can excite the amplitudes of the two modes by slightly different amounts. Since the periods are then incommensurate, small differences can lead to sizeable numerical changes. Bimodal coherence exhibits features similar to chaotic behavior, in that it is very sensitive to small changes in parameters and initial conditions.

To determine the behavior of a particular simulation, one can examine the time evolution of the components of average neutrino vector $\langle\vec{v}(t)\rangle=\left\langle\left(v_{1}(t), v_{2}(t), v_{3}(t)\right)\right\rangle$. However, the single or bimodal nature of coherent oscillations is often better displayed as the three-dimensional orbit of $\langle\vec{v}(t)\rangle$. In the current work, bimodal oscillations were evident in almost all the simulations in the irregular region. Figure 1 shows an example. In a few of the simulations, the orbits appear irregular: it is impossible to decide if only two modes occur. It may be that some irregular behavior is not strictly bimodal coherence due to the expansion of the Universe or other effects not included in the analysis of ref. 22.

Figure 2 shows our results in the $\sin ^{2} 2 \theta-\Delta$ plane. The region labelled 'weak bimodal,' classified as 'residual self-maintained coherence' in ref. [12], is bimodal coherence with one mode having a much smaller amplitude than the other. It is almost 
self-maintained coherence but exhibits irregular-type behavior over sufficiently long time scales. Simulation for $\Delta<-10^{-4} \mathrm{eV}^{2}$ is prohibitive in computer time, but we can determine the behavior by other arguments. The essential physics is unchanged from $\Delta=-10^{-4} \mathrm{eV}^{2}$ to $\Delta=-1 \mathrm{eV}^{2}$, so irregular behavior should appear in this region. For $\Delta<-1 \mathrm{eV}^{2}$, vacuum oscillation effects dominate over the nonlinear forward scattering of neutrinos, and so vacuum behavior is expected. This region is labelled 'vacuum decoherence' in Figure 2. The production of excess neutrinos quickly decoheres and neutrinos become vacuum mass eigenstates.

For positive $\Delta$, the sum of the excess of electron and muon neutrinos is insensitive to the effects of neutrino oscillations [16]. The same is true of the tau-neutrino excess. For negative $\Delta$, we find similar results. Hence, our new curves for $\left\langle v_{0}(t)\right\rangle$ and $\left\langle v_{4}(t)\right\rangle$ exhibit little dependence on $\Delta$ and $\sin ^{2} 2 \theta$. They are essentially identical to the dashed-dotted and dashed curves in Figure 3 of ref. [16]. Likewise, the final energy distributions for the sum of the excess of electron and muon neutrinos and for the tauneutrino excess are insensitive to $\Delta$ and $\sin ^{2} 2 \theta$ and are given by the dashed-dotted and dashed curves in Figure 1 of ref. [16].

In contrast, the remaining three variables $\left\langle v_{1}(t)\right\rangle,\left\langle v_{2}(t)\right\rangle$, and $\left\langle v_{3}(t)\right\rangle$ depend on $\Delta$ and $\sin ^{2} 2 \theta$. The difference $\left\langle v_{1}(t)\right\rangle$ between the excesses of electron and muon neutrinos starts out at zero at $t=0.08$ seconds when $T=3.0 \mathrm{MeV}$, rises as excess electron neutrinos are produced, and typically declines after the instability time as many electron neutrinos oscillate into muon neutrinos. At the instability time and thereafter, $\left\langle v_{2}(t)\right\rangle$ and sometimes $\left\langle v_{3}(t)\right\rangle$ become significantly different from zero. The sizeable conversion of electron neutrinos into muon neutrinos throughout the $\Delta<0$ region is the result of a new resonance conversion mechanism [12] driven by the imaginary part of the off-diagonal component of the neutrino density matrix, which represents the difference between the third components of the average vectors for neutrinos and antineutrinos.

Figure 3 displays $\left\langle v_{1}(t)\right\rangle,\left\langle v_{2}(t)\right\rangle$ and $\left\langle v_{3}(t)\right\rangle$ for the case $\Delta=-10^{-5} \mathrm{eV}^{2}$ and $\sin ^{2} 2 \theta=10^{-3}$, a point lying in the irregular region of Figure 2. Figure 4 displays the same three quantities for the case $\Delta=-10^{-6} \mathrm{eV}^{2}$ and $\sin ^{2} 2 \theta=10^{-8}$, which lies in the weak-bimodal region. Rapid fluctuations occur that are not visible on the scale of 
Figure 4. In Figure 5, we plot the components over a short time interval at $t \approx 0.94$ seconds. The corresponding orbit is shown in Figure 6. Since irregular behavior occurs throughout most of the region with $\Delta<-10^{-6} \mathrm{eV}^{2}$ and vectors undergo sizeable unpredictable fluctuations, it makes little sense to plot the final production profiles for $\vec{v}$ as a function of energy.

For $-10^{-4} \mathrm{eV}^{2}<\Delta$, ANME configurations at early times are flavor eigenstates with an excess of electron neutrinos, so the first component of $\vec{v}(E, t)$ is the largest. At later times, ANME configurations undergo a $180^{\circ}$ rotation in three-space. If the nonlinear neutrino-neutrino interactions were absent, then little conversion of electron neutrinos into muon neutrinos would occur when the rotation is non-adiabatic. However, after the rotation occurs, the neutrino-neutrino interactions render the system unstable and a large conversion of electron neutrinos into muon neutrinos takes place, regardless of the rotation rate [12, 14]. The instability time is expected to occur when the vacuum and $\mathrm{CP}$-conserving electron-neutrino terms become equal [12, 14], i.e., when

$$
|\Delta| \cos (2 \theta) /(2 \bar{E}) \approx\left|V_{C P+}\right|=2 \sqrt{2} G_{F} \bar{E}\left(\rho_{e^{-}}+p_{e^{-}}+\rho_{e^{+}}+p_{e^{+}}\right) / M_{W}^{2},
$$

where $\bar{E}$ is the average neutrino energy, $G_{F}$ is Fermi coupling constant, $M_{W}$ is the mass of the $W$ boson, and $\rho_{e^{ \pm}}$and $p_{e^{ \pm}}$are, respectively, the energy densities and pressures of the background positrons and electrons.

When $\theta$ is small so that $\cos 2 \theta \approx 1$, we find that Eq. (四) predicts instability times of $1.1,0.5$, and 0.23 seconds for $\Delta=-10^{-7} \mathrm{eV}^{2},-10^{-6} \mathrm{eV}^{2}$ and $-10^{-5} \mathrm{eV}^{2}$, respectively. In our numerical simulations, the corresponding instability times are found to be $\sim 1.0, \sim 0.45$ and $\sim 0.2$ seconds, in good agreement with the theoretical expectations.

Similarly, when $\sin ^{2} 2 \theta$ is near one we again find good agreement. For example, when $\sin ^{2} 2 \theta=0.49$, the instability times occur in simulations at $\sim 0.55, \sim 0.25$ and $\sim 0.12$ seconds for $\Delta=-10^{-6} \mathrm{eV}^{2},-10^{-5} \mathrm{eV}^{2}$ and $-10^{-4} \mathrm{eV}^{2}$, respectively. The corresponding values from Eq. (四) are 0.52, 0.24 and 0.11 seconds.

For $\Delta<-10^{-4} \mathrm{eV}^{2}$, the instability time as determined by Eq. (1) occurs before production begins. Hence, the instability appears as soon as the electron-neutrino 
excess becomes sufficiently large to render the neutrino-neutrino interactions important. This happens soon after production begins. The instability disrupts the system and leads to the irregular behavior seen in this region in Figure 2.

Other features of the neutrino oscillations are similar to the positive- $\Delta$ case [14]. In particular, the CP-suppression mechanism is still operative. In the regions of coherent oscillations, the $\mathrm{CP}$ asymmetry of the excess can sometimes reach the $1 \%$ level, but this is insufficient to affect big-bang nucleosynthesis at a level observable with current technology. The evolutions of the antineutrino excesses are almost identical to those of the corresponding neutrinos, so we have not displayed them.

To summarize, we have obtained the complete phase diagram for neutrino oscillations for the case of an inverted neutrino-mass hierarchy. Five different behaviors are observed: smooth evolution, self-maintained coherence, bimodal coherence, irregular behavior, and vacuum decoherence. Throughout most of the large- $|\Delta|$ region, irregular coherent oscillations are seen.

\section{Acknowledgments}

This work is supported in part by the United States Department of Energy (grant numbers DE-FG02-91ER40661 and DE-FG02-92ER40698), by the Alexander von Humboldt Foundation, and by the PSC Board of Higher Education at CUNY.

\section{References}

1. See, for example, E.W. Kolb and M.S. Turner, The Early Universe (AddisonWesley, Redwood City, CA, 1990).

2. A. D. Dolgov and M. Fukugita, Phys. Rev. D46 (1992) 5378.

3. S. Dodelson and M. S. Turner, Phys. Rev. D46 (1992) 3372;

B. Fields, S. Dodelson and M. S. Turner, Phys. Rev. D47 (1993) 4309.

4. A. D. Dolgov, Sov. J. Nucl. Phys. 33 (1981) 700.

5. L. Stodolsky, Phys. Rev. D36 (1987) 2273.

6. P. Langacker, S. T. Petcov, G. Steigman, and S. Toshev, Nucl. Phys. B282 
(1987) 589 .

7. D. Notzold and G. Raffelt, Nucl. Phys. B307 (1988) 924.

8. M. J. Savage, R. A. Malaney and G. M. Fuller, Astrophys. J. 368 (1991) 1.

9. K. Enqvist, K. Kainulainen and J. Maalampi, Nucl. Phys. B349 (1991) 754.

10. K. Enqvist, K. Kainulainen and M. Thomson, Nucl. Phys. B373 (1992) 498.

11. V. A. Kostelecký, J. Pantaleone and S. Samuel, Phys. Lett. B315 (1993) 46.

12. V. A. Kostelecký and S. Samuel, Phys. Lett. B318 (1993) 127.

13. X. Shi, D. N. Schramm and B. D. Fields, Phys. Rev. D48 (1993) 2563.

14. V. A. Kostelecký and S. Samuel, Phys. Rev. D49 (1994) 1740.

15. B. H. J. McKellar and M. J. Thomson Phys. Rev. D49 (1994) 2710.

16. V. A. Kostelecký and S. Samuel, Phys. Rev. D52 (1995) 3184.

17. G. M. Fuller, J. R. Primack, Y.-Z. Qian, Phys. Rev. D52 (1995) 1288.

18. D. O. Caldwell and R. N. Mohapatra Phys. Lett. B354 (1995) 371.

19. G. Raffelt and J. Silk, Phys. Lett. B366 (1996) 429.

20. S. Samuel, Phys. Rev. D48 (1993) 1462.

21. V. A. Kostelecký and S. Samuel, Phys. Rev. D52 (1995) 621.

22. S. Samuel, Bimodal Coherence in Dense Self-Interacting Neutrino Gases, CCNY-HEP-95/5 (July, 1995), to appear in Phys. Rev. D. 


\section{Figure Captions}

Figure 1: An orbit in the irregular region for $\sin ^{2} 2 \theta=10^{-7}, \Delta=-10^{-5} \mathrm{eV}^{2}$ near $t=0.42$ seconds. The first mode involves motion around a circle. The second mode rotates the circle. Only a few periods of the first mode are included. When the orbit is displayed over longer time intervals, the bimodal coherence produces a figure which looks like a ball of wool.

Figure 2: The phase diagram for neutrino flavor oscillations for an inverted neutrinomass hierarchy.

Figure 3: Time development of the nonequilibrium distortions for the case $\Delta=-10^{-5}$ $\mathrm{eV}^{2}$ and $\sin ^{2} 2 \theta=10^{-3}$. (a) The quantity $\left\langle v_{1}(t)\right\rangle$ is shown as a function of time. (b) The quantity $\left\langle v_{2}(t)\right\rangle$ is shown as a function of time. Note the change of vertical scale relative to (a). (c) The quantity $\left\langle v_{3}(t)\right\rangle$ is shown as a function of time. Note the change of vertical scale relative to (a).

Figure 4: Time development of the nonequilibrium distortion for the case $\Delta=-10^{-6}$ $\mathrm{eV}^{2}$ and $\sin ^{2} 2 \theta=10^{-8}$. (a) The quantity $\left\langle v_{1}(t)\right\rangle$ is shown as a function of time. (b) The quantity $\left\langle v_{2}(t)\right\rangle$ is shown as a function of time. (c) The quantity $\left\langle v_{3}(t)\right\rangle$ is shown as a function of time.

Figure 5: Time development of the nonequilibrium distribution for the case in Figure 4, with $\Delta=-10^{-6} \mathrm{eV}^{2}$ and $\sin ^{2} 2 \theta=10^{-8}$. The components have been scaled by a factor of about 250 for clarity.

Figure 6: Orbit for the case $\Delta=-10^{-6} \mathrm{eV}^{2}$ and $\sin ^{2} 2 \theta=10^{-8}$. The three quantities displayed in Figure 5 are combined into a vector and its trajectory is plotted in three-dimensional space. 
This figure "figure1.gif" is available in "gif" format from: http://arxiv.org/ps/hep-ph/9610399v2 


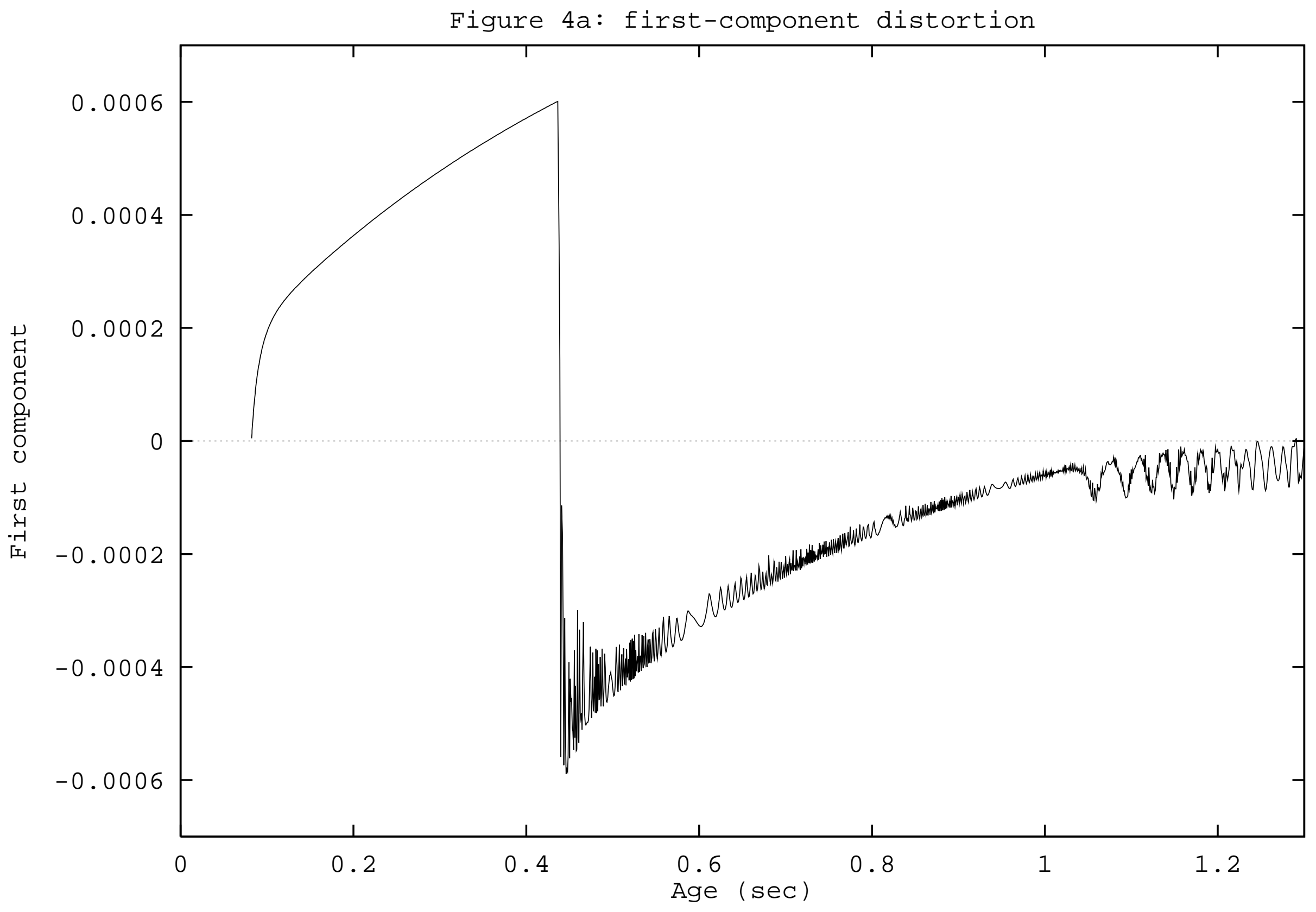


This figure "figure2.gif" is available in "gif" format from: http://arxiv.org/ps/hep-ph/9610399v2 


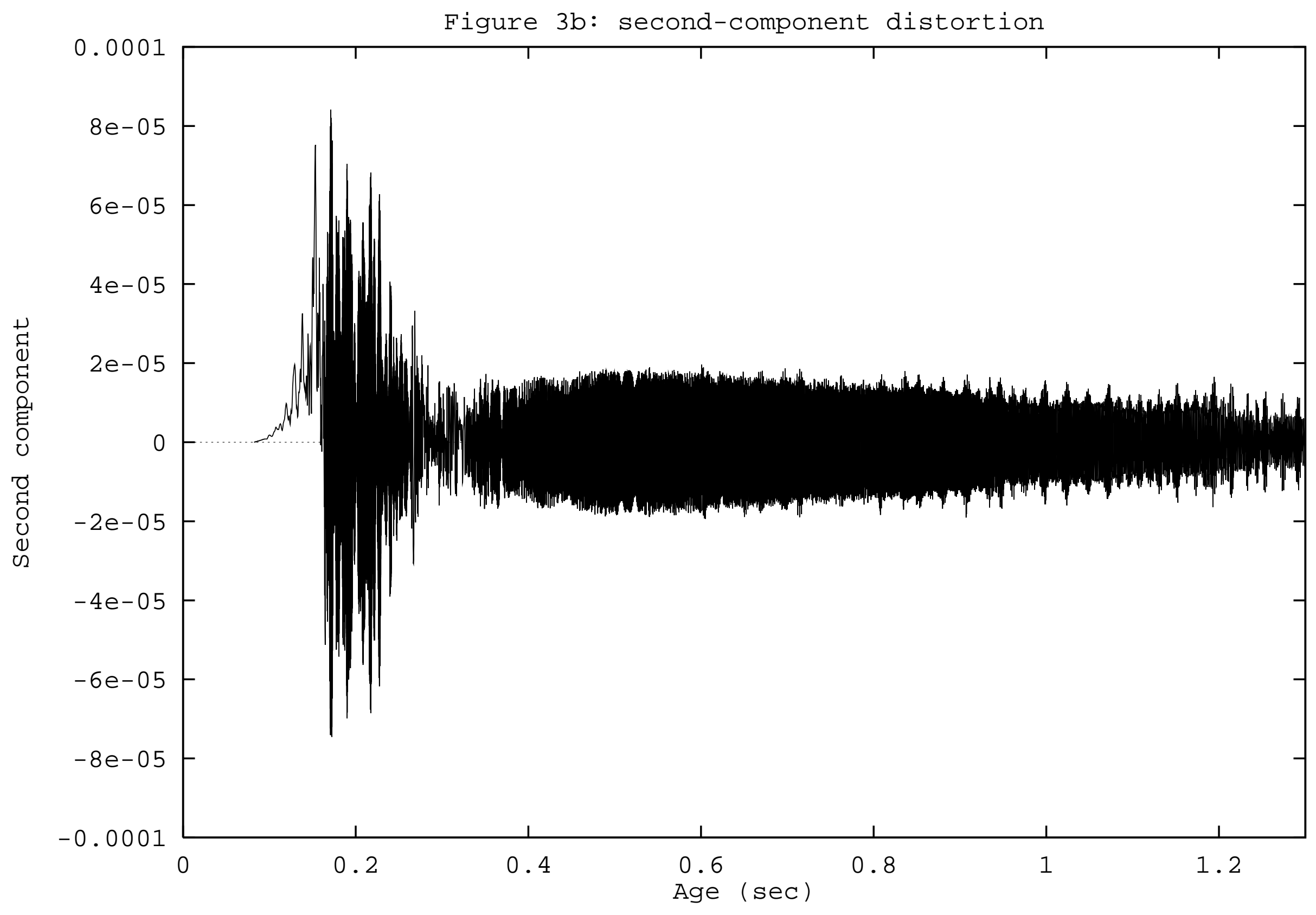




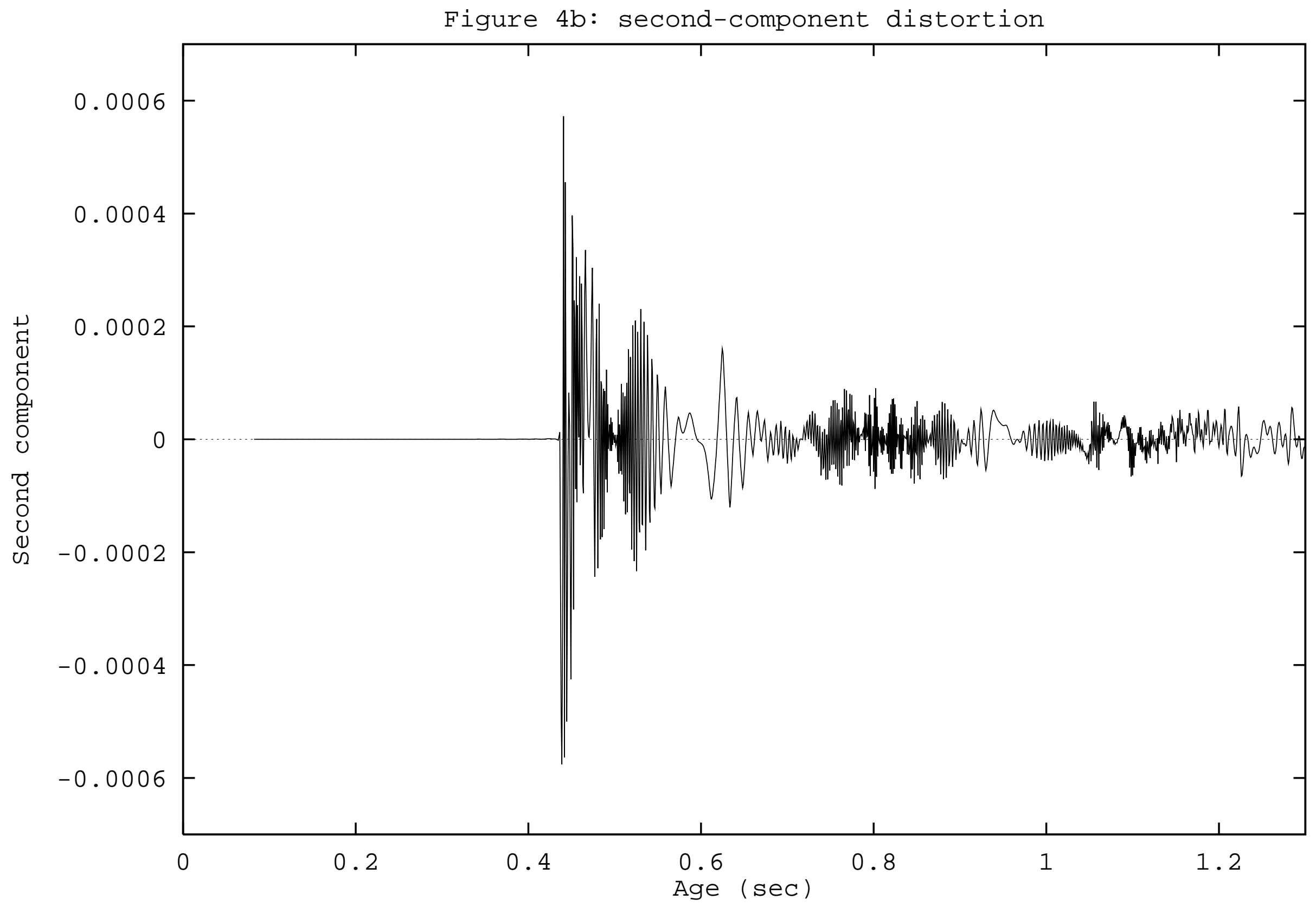




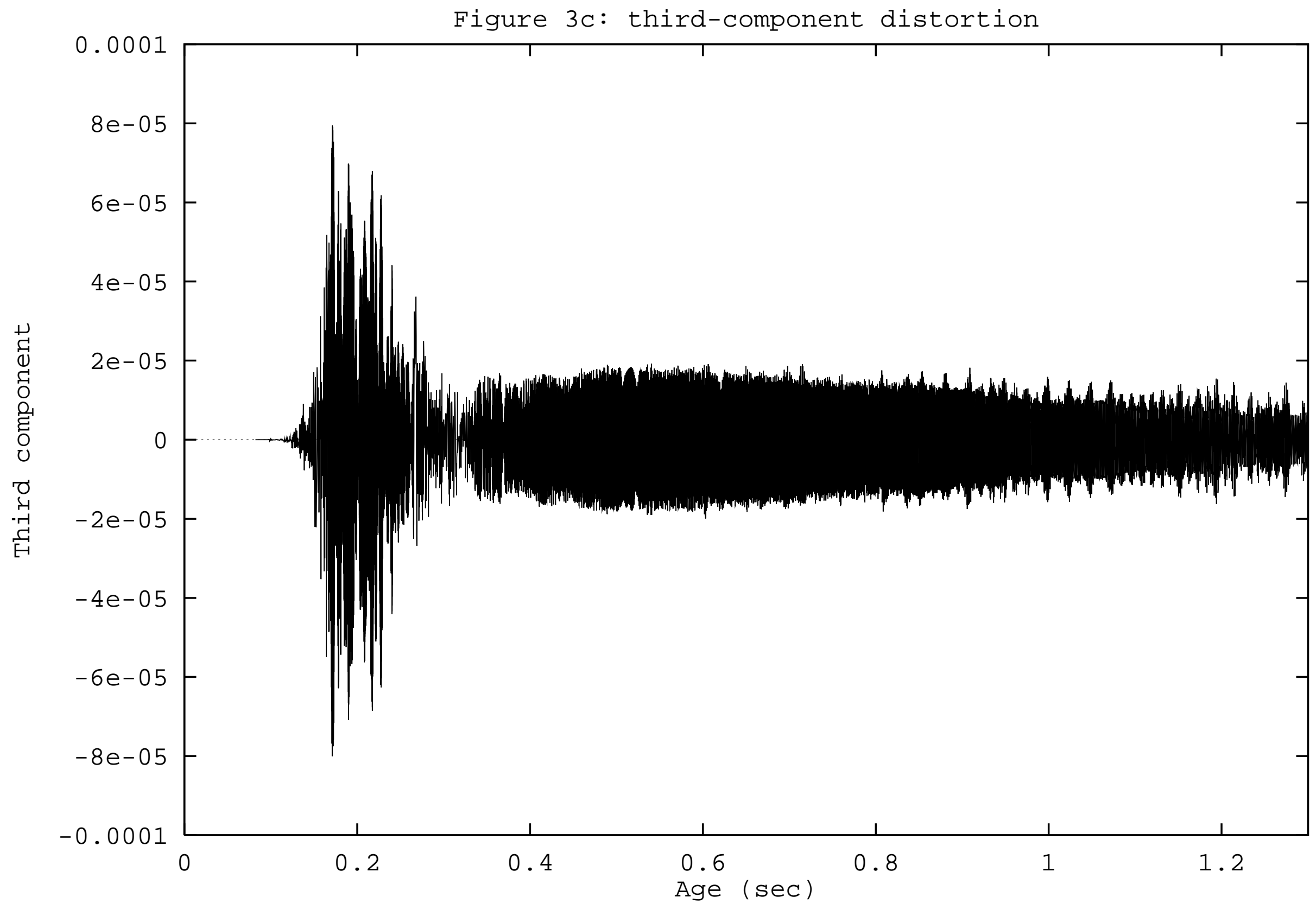




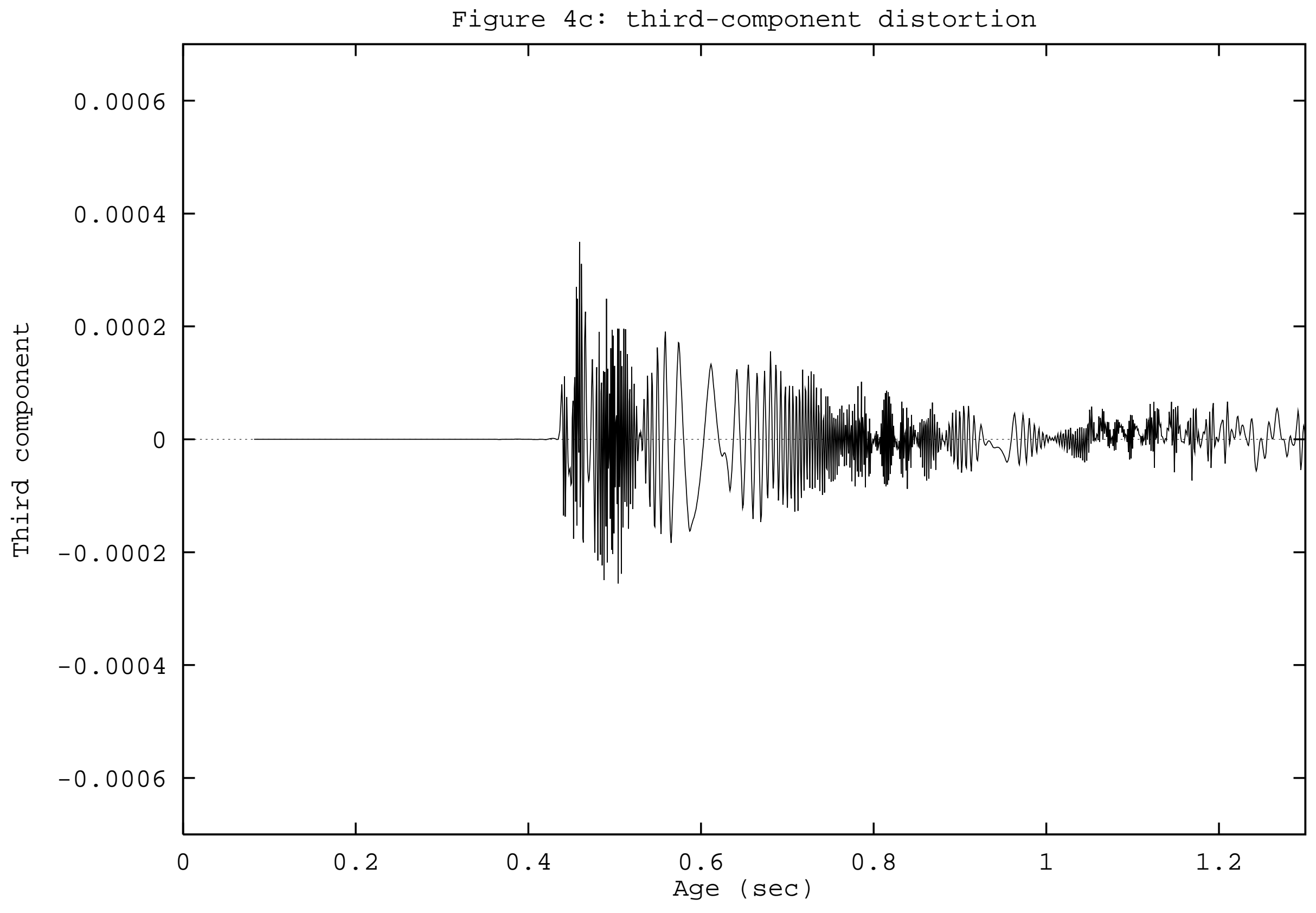


This figure "figure5.gif" is available in "gif" format from: http://arxiv.org/ps/hep-ph/9610399v2 
This figure "figure6.gif" is available in "gif" format from: http://arxiv.org/ps/hep-ph/9610399v2 


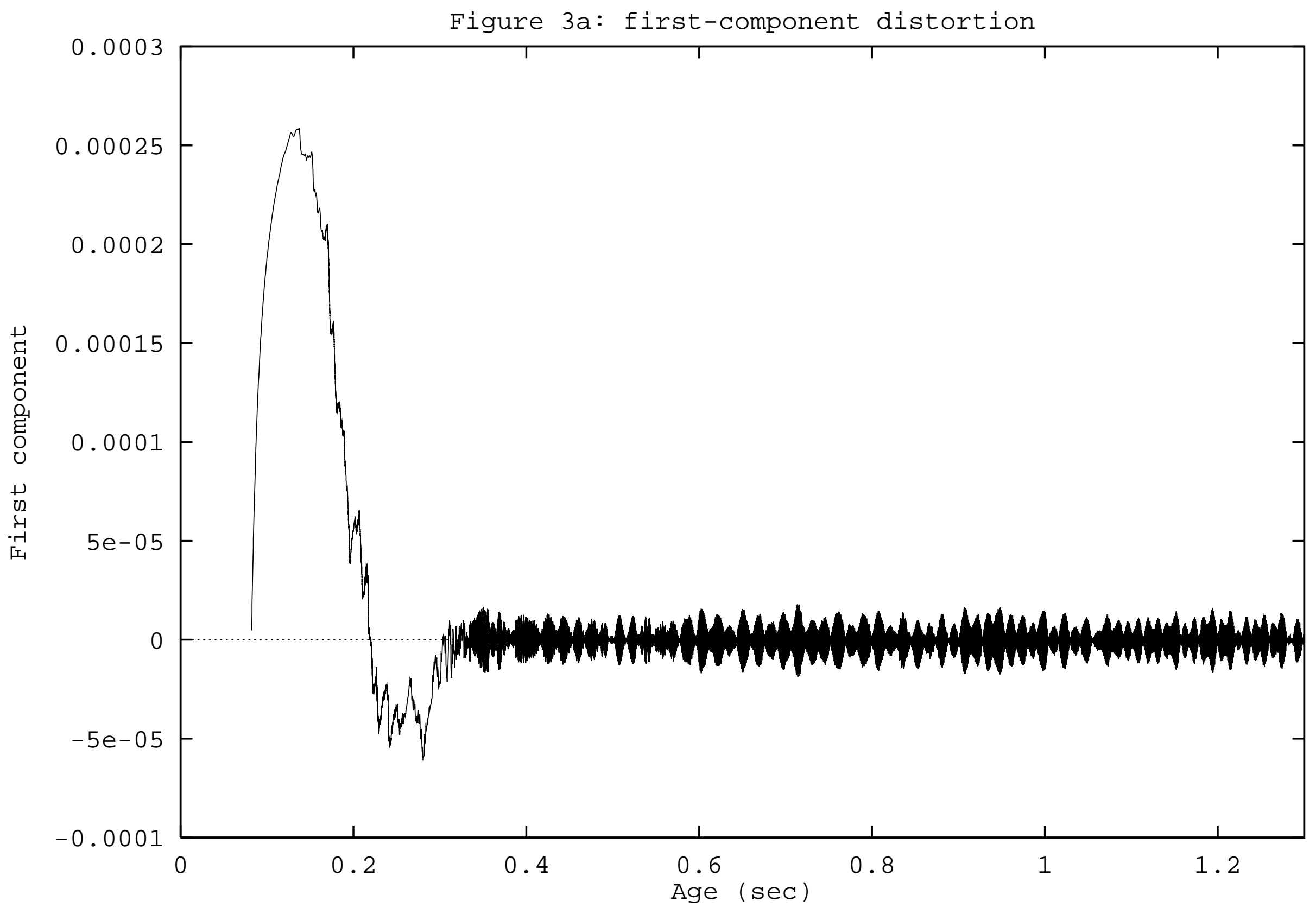

